\title{
Impacto da Desidratação na Geração de Força de Atletas de Arco e Flecha Durante Competição Indoor e Outdoor
}

\author{
Impact of Dehydration in the Strength Generation of \\ Archery Athletes During Indoor and Outdoor Competition
}

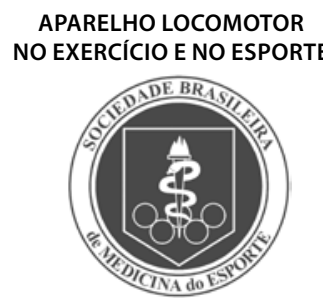

Artigo Original

\begin{abstract}
Vanessa de Brito Reis ${ }^{1}$
Marília Cerqueira Leite Seelaender ${ }^{1}$ Luciana Rossi

1. Instituto de Ciências Biomédicas da Universidade de São Paulo, São Paulo. 2. Centro Universitário São Camilo, São Paulo.
\end{abstract}

Endereço para correspondência: Rua Augusto Viana de Castelo, 108 Mandaqui - 02471-020 - São Paulo, SP. E-mail: vanessa.breis@yahoo.com.br

\begin{abstract}
RESUMO
Introdução: O arco e flecha é um esporte estático que requer força e resistência da parte superior do corpo, sendo que a habilidade do arqueiro é definida pela capacidade de atirar a flecha no alvo em um intervalo de tempo com precisão. A contração muscular gerada durante a execução do gesto esportivo propicia o aumento da temperatura corporal, da oxidação de glicose e da sudorese, podendo esta ser potencializada pelas condições climáticas às quais o atleta é exposto. Todos estes fatores alteram a percepção de esforço e a própria geração de força, impondo um estresse negativo à performance. Objetivos: Avaliar e relacionar o estado de hidratação de atletas de arco e flecha com alterações fisiológicas e de força intervenientes no rendimento competitivo. Métodos: Participaram 11 arqueiros em duas competições distintas (indoor e outdoor). Foram mensurados parâmetros relacionados ao estado de hidratação, glicemia, percepção de esforço subjetivo e alteração na força de preensão palmar. Resultados: Os atletas analisados apresentaram alterações significativas nos parâmetros relacionados ao estado hídrico em diferentes dias, provavelmente relacionadas às condições climáticas adversas na competição outdoor $\left(34^{\circ} \mathrm{C}\right.$ e $60 \%$ URA) em relação à competição indoor $\left(22^{\circ} \mathrm{C}\right.$ e $90 \%$ URA). O mesmo não foi observado para a glicemia. Conclusão: Este trabalho é o primeiro a comprovar a relação direta entre fatores climáticos e de rendimento em arqueiros de alto nível durante evento competitivo, assim como a evidenciar quais métodos não invasivos seriam os mais indicados para a avaliação do impacto destas alterações.
\end{abstract}

Palavras-chave: arco e flecha, desidratação, preensão palmar, rendimento.

\begin{abstract}
Introduction: Archery is a static sport that requires strength and resistance from the upper part of the body, and the skill of the archer is defined by the capacity to shoot the arrow on the target in an interval of time with accuracy. The muscle contraction generated during the performance of the sports gesture causes the increase in body temperature, glucose oxidation and the sweat loss, being able to be boosted by the climate conditions to which the athlete is exposed. These factors combined modify the effort perception and the proper strength generation, imposing hence stress negative to performance. Objectives: To evaluate and to relate the hydration status of archery athletes to intervening physiological and strength alterations in the competitive performance. Methods: 11 archers participated in two distinct competitions (indoor and outdoor). Parameters related to the hydration status, glycemia, subjective perceived exertion and alteration in handgrip were measured. Results: The analyzed athletes presented significant alterations in the parameters related to their hydration on different days, probably related to the adverse weather conditions in the outdoor events $\left(34^{\circ} \mathrm{C}\right.$ and $\left.60 \%\right)$. The same situation was not observed for glycemia. Conclusion: This investigation is a pioneer in proving the straight relation between weather conditions and performance in high-level archers during competitive events, as well as evidencing which non-invasive methods would be recommended to assessment of the impact of these alterations.
\end{abstract}

Keywords: archery, dehydration, handgrip, performance.

\section{INTRODUÇÃO}

O arco e flecha é considerado um esporte estático que requer força e resistência da parte superior do corpo, particularmente nos braços, cintura e ombro. A habilidade do arqueiro é definida pela capacidade de atirar a flecha no alvo em um determinado intervalo de tempo com máxima precisão(1).
Existem competições nos mais diversos âmbitos, sejam estudantis, regionais e até jogos olímpicos e campeonatos mundiais ${ }^{(2)}$. Estas costumam durar, em média, de cinco a sete horas, com intervalo de 30 minutos a cada duas-três horas de competição. As modalidades mais praticadas são: 1) Indoor: competição realizada em ambientes fechados à distâncias entre 18 e 30 metros, originária de países 
com invernos rigorosos, onde a prática ficava suspensa por grandes períodos; e 2) Outdoor: a mais tradicional e principal forma de competição internacional, realizada com disparos a longas distâncias, entre 30 e 90 metros $^{(3)}$.

No arco e flecha, como em muitos outros esportes, a proximidade da competição é motivo de ansiedade, que, dependente do nível competitivo, pode impor um grande estresse, fator este interveniente no grau de precisão do atleta e, portanto, determinante no desempenho ${ }^{(4)}$. O acúmulo e interação de fatores estressores nos períodos de treinamento e intervalos podem auxiliar na explicação de como ocorrem as alterações no desempenho atlético ${ }^{(5)}$, estresse este expresso de diferentes formas em cada indivíduo. No dia anterior à competição e nos momentos que a precedem, estes comportamentos, muitas vezes despercebidos, podem trazer prejuízos de grande escala para o atleta, tanto pelos aspectos psicológicos e cognitivos, como pelos aspectos físicos, refletidos em tremores nas mãos, aumento nos batimentos cardíacos e outras alterações importantes como a transpiração excessiva ${ }^{(4)}$, sendo esta responsável por impor um quadro de desidratação no atleta que, por fim, pode acarretar em diminuição na função neuropsicológica e na estabilidade postural(6,7). A desidratação pode ainda afetar a função cognitiva, compreendida como as fases pelas quais as informações são processadas: percepção, aprendizagem, memória, atenção, vigilância, raciocínio e solução de problemas, incluindo funcionamento psicomotor (tempo de reação, tempo de movimento e velocidade de desempenho) $)^{(8,9)}$, funções essenciais ao arqueiro. Tais prejuízos na cognição são resultantes da hipovolemia e de uma subsequente hipoperfusão cerebral (figura 1). Embora evidentes durante o progresso de desidratação moderada a severa, as manifestações da desidratação leve na função cognitiva não foram totalmente exploradas ${ }^{(10)}$.

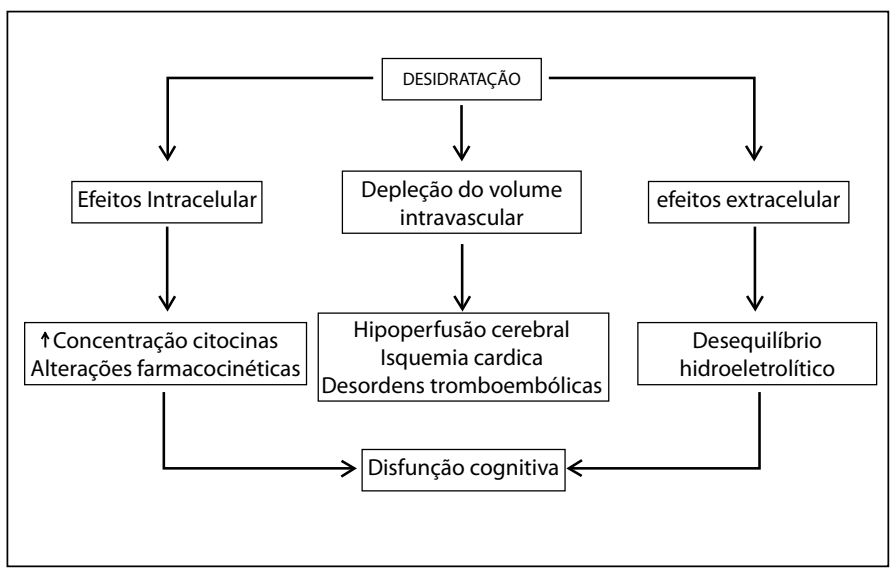

Adaptado de Wilson e Morley, 2003.

Figura 1. Patofisiologia da disfunção cognitiva na desidratação moderada e severa.

Mesmo atletas com um baixo gasto calórico durante a atividade, mas que competem em áreas abertas como o arco e flecha, também podem apresentar um risco de desidratação, afetando a performance ${ }^{(11)}$.

Além do estado de hidratação para manutenção da função cognitiva e adequada contração muscular, tem-se que o carboidrato é o principal combustível utilizado na prática de arco e flecha, e sua disponibilidade para os músculos constitui um fator limitante no desempenho do atleta, podendo levar a um estado de fadiga muscular precoce $^{(12,13)}$. A fadiga muscular pode ser identificada pela falha em manter o nível desejado de trabalho ou performance, e pode estar relacionada com algumas variáveis, tais como massa muscular envolvida durante a atividade física, intensidade da contração muscular, velocidade do movimento executado, taxa de amplitude do movimento, frequência de contração, relaxamento muscular e quantidade de substrato energético disponível(14).
A restrição no consumo de fontes de carboidratos pode alterar a expressão serotoninérgica e consequentemente afetar aspectos psicológicos funcionais ${ }^{(15,16)}$, além da própria redução na concentração de glicose sanguínea e do glicogênio muscular e hepático. Além disso, o aporte adequado deste substrato é indispensável para a síntese, ação e metabolismo de neurotransmissores ${ }^{(9,17)}$. Adicionalmente, estas alterações podem afetar as funções do sistema nervoso central, alterando o estado de humor, motivação, transformação das informações recebidas, percepção de esforço e/ou excitabilidade do córtex motor, prejudicando o desempenho durante o exercício(16).

Não só a adequação da dieta está relacionada com a melhoria do rendimento esportivo, mas também a adequação do estado psicológico, refletindo na manutenção da qualidade de vida do atleta. Lane $^{(18)}$ relacionou o estado de humor depressivo pré-competição com a performance cognitiva, na qual este promoveu um quadro de diminuição do vigor, aumento de estado de confusão, tensão, fadiga e nervosismo.

Verifica-se ausência de trabalhos científicos correlacionando estado nutricional ao rendimento de atletas de arco e flecha, expondo lacunas entre os conhecimentos específicos sobre o esporte, principalmente em relação ao perfil antropométrico e necessidades nutricionais para melhor performance. O objetivo deste trabalho compreende avaliar e discutir o impacto no estado hídrico no rendimento de arqueiros em competição indoor e outdoor em relação à geração de força muscular de membro superior com finalidade de expor e delinear futuras estratégias nutricionais relacionadas à melhora no rendimento esportivo.

\section{MÉTODOS}

O estudo foi realizado com 13 arqueiros, 11 do sexo masculino e dois do feminino, pertencentes ao quadro de atletas competitivos da equipe de um clube de São Paulo, todos em fase de competição na modalidade outdoor (30 a 90 metros de distância) e indoor (30 metros). O projeto foi previamente aprovado pelo Comitê de Ética em Pesquisa (n० 148/08) e todos os participantes assinaram um termo de consentimento esclarecido.

A avaliação antropométrica constituiu na obtenção do peso $(\mathrm{kg})$ através de balança digital da marca Plenna ${ }^{\circledR}$, modelo TIN 126, com precisão de $0,1 \mathrm{~kg}$ e para a estatura $(\mathrm{m})$, foi empregado estadiômetro da marca Sanny ${ }^{\circledR}$, com precisão de $1 \mathrm{~mm}$ e comprimento máximo de $210 \mathrm{~cm}$. O avaliado foi posicionado no plano de Frankfurt para adequada mensuração ${ }^{(19)}$. Com os valores de estatura e de peso foi calculado o índice de massa corpórea (IMC), através da relação IMC = peso/estatura² sendo o estado nutricional classificado segundo a OMS ${ }^{(19)}$.

Conforme padronização ${ }^{(19)}$, foram mensuradas as seguintes dobras cutâneas no hemicorpo direito, através do emprego do compasso da marca Sanny ${ }^{\circledR}$ modelo Clínico, com precisão $\pm 1 \mathrm{~mm}$ : bíceps $(\mathrm{DB})$, tríceps (DT), subescapular (DSe), torácica (DTx), axilar média (DAx), suprailíaca (DSi), abdominal (DA), coxa (DC). Com os valores obtidos, foi calculado o percentual de gordura (\%G) através de equação antropométrica específica para atletas do sexo feminino de Jackson et al.(20) e sexo masculino de Jackson e Pollock ${ }^{(21)}$. O percentual de gordura foi classificado de acordo com os valores de referência propostos por Lohman et al. ${ }^{(22)}$.

Para determinação do estado de hidratação, foi avaliada a perda hídrica total durante a competição, em que os participantes foram pesados antes (peso inicial - Pi) e após a prova (peso final - Pf). Estes valores foram utilizados para o cálculo da taxa de sudorese (TS em L/h) através da fórmula: TS = (Pi - Pf) / tempo total de atividade física ${ }^{(23)}$. A perda de peso corporal foi corrigida pelo volume de líquidos e de alimentos ingeridos durante o período de competição, conforme descrito por Shirreffs em $2003^{(24)}$. Nos dias de competição, tanto a temperatura 
ambiente como a umidade relativa do ar (URA) foram avaliadas por um termo higrômetro marca Minipa modelo MT-240.

Na avaliação da força muscular foi empregado o teste de preensão palmar que segundo o manual do ACSM(25), é um biomarcador confiável e aplicável em situações de coleta de campo. Para tanto, antes e após a competição, o indivíduo foi orientado para ficar em pé com um dinamômetro digital DayHome, modelo EH 101, paralelo ao eixo do corpo aproximadamente ao nível da cintura com o antebraço ao nível da coxa. A seguir, foi requisitado realizar a preensão com força máxima; após três medidas, a de maior magnitude foi anotada. O procedimento se repetiu para o outro braço. Para avaliação da força de preensão total, os valores de força de membro direito e esquerdo foram somados, adotando-se este procedimento para minimizar interferência na força de preensão individual devido dominância do membro(25).

Foi empregado um analisador portátil de glicose sanguínea da marca Accu-Chek ${ }^{\circledR}$ Active, para avaliação bioquímica, que permitiu a determinação da glicemia em cinco segundos. A concentração de glicose sanguínea foi medida antes e no final da competição.

A avaliação da percepção subjetiva de esforço foi realizada antes e após a competição empregando-se a escala numérica de 15 pontos proposta por Borg, e os valores foram correlacionados com a autopercepção de esforço físico ${ }^{(26)}$. Este instrumento é amplamente utilizado nos esportes e programas de treinamento físico, por correlacionar bem com o consumo de oxigênio $\left(\mathrm{VO}_{2}\right)$ e frequência cardíaca ${ }^{(27)}$.

Os dados foram expressos em medidas de tendência central e variabilidade. Para determinação das diferenças intragrupos entre as médias foi empregado teste $t$ pareado e entre grupos teste $t$ não pareado, com probabilidade menor que 5\% $(p<0,05)$ para detecção da hipótese nula.

\section{RESULTADOS}

Os atletas possuíam, em média, 34,1 \pm 7,2 anos de idade; 86,0 \pm $23,5 \mathrm{~kg}$ de massa corporal; $1,74 \pm 0,09 \mathrm{~m}$ de estatura, IMC de 28,08 \pm $6,30 \mathrm{~kg} / \mathrm{m}^{2}$ e percentual de gordura corporal de $21,2 \pm 4,4 \%$. Quanto ao estado nutricional, segundo o IMC, a amostra apresenta sobrepeso ${ }^{(19)}$, com percentual de gordura acima da média(22).

$\mathrm{Na}$ avaliação do estado de hidratação na competição indoor, a temperatura ambiente encontrava-se em $22^{\circ} \mathrm{C}$, com a umidade relativa do ar (URA) de 90\%. Neste dia, a taxa média de sudorese foi de 1,5 $\pm 1,4 \mathrm{~L} / \mathrm{h}$ com um percentual médio de perda de peso de 1,4 $\pm 0,5 \%$.

No dia de competição outdoor, a temperatura encontrava-se em $34^{\circ} \mathrm{C}$, com URA de $60 \%$. A taxa de sudorese foi de $2,2 \pm 1,3 \mathrm{~L} / \mathrm{h}$ e perda de peso corporal de $2,1 \pm 0,7 \%$, ambos os valores significativamente maiores $(p<0,05)$ que no dia de competição indoor. A figura 2 mostra a variação do peso pré-competição com os valores de peso pós-competição, nos dois dias de prova analisados. Ambas as provas tiveram duração média de cinco horas cada.

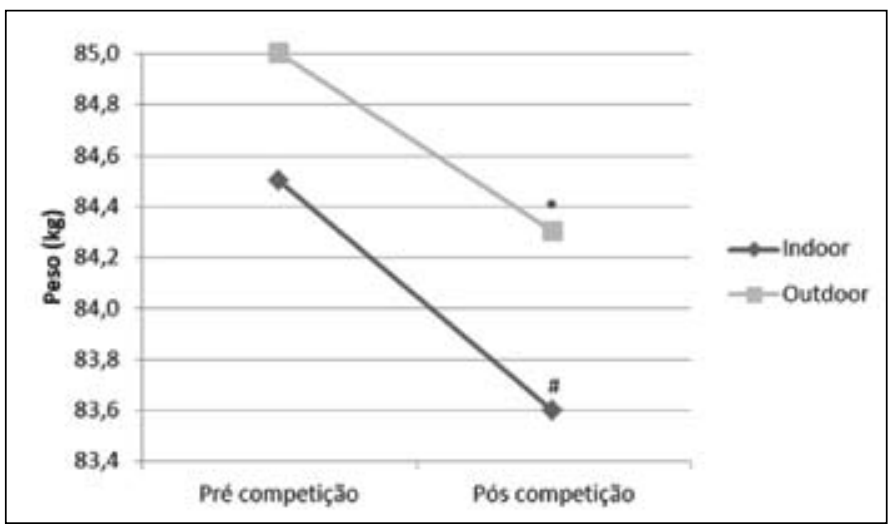

Figura 2. Variação média do peso durante as competições indoor e outdoor de arco e flecha (\# $\left.{ }^{*} p<0,05\right)$.
Quando analisado o consumo hídrico ad libitum durante toda a prova nos dois dias, verificou-se uma diferença estatisticamente significativa $(p<0,005)$ entre consumo hídrico na competição indoor, valor médio de 0,6 $\pm 03 \mathrm{~L}$ e a competição outdoor de 1,0 \pm 0,2L. A ingestão de líquido foi significativamente menor que a perda hídrica nos dois dias de competição, conforme mostra a figura 3 .

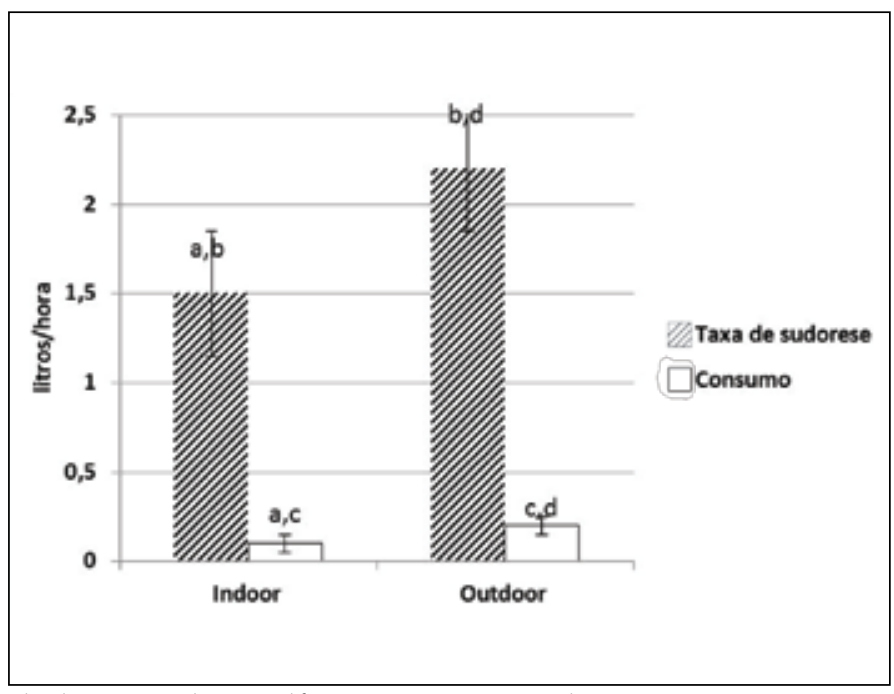

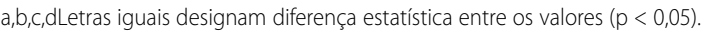

Figura 3. Relação entre o consumo hídrico e a taxa de sudorese nas competições indoor e outdoor de arco e flecha.

Tanto nos dias de competição indoor como outdoor, a glicemia final tendeu a concentrações maiores do que as iniciais, embora não estatisticamente significativos. Na competição indoor, os atletas apresentaram inicialmente uma glicemia de $92,6 \pm 12,4 \mathrm{mmol} / \mathrm{L}$ e ao final $97,1 \pm 12,3 \mathrm{mmol} / \mathrm{L}$. Na outdoor, os valores iniciais e finais foram, respectivamente, $98,5 \pm 13,1 \mathrm{mmol} / \mathrm{L}$ e $101,3 \pm 16,4 \mathrm{mmol} / \mathrm{L}$.

Os valores de preensão palmar foram estatisticamente diferentes entre o período pré e pós-competição indoor $(p<0,005)$ e outdoor ( $p$ $<0,05)$ como mostra a figura 4. Entretanto, os valores antes e depois da competição apresentaram resultados diferenciados entre os dois dias.

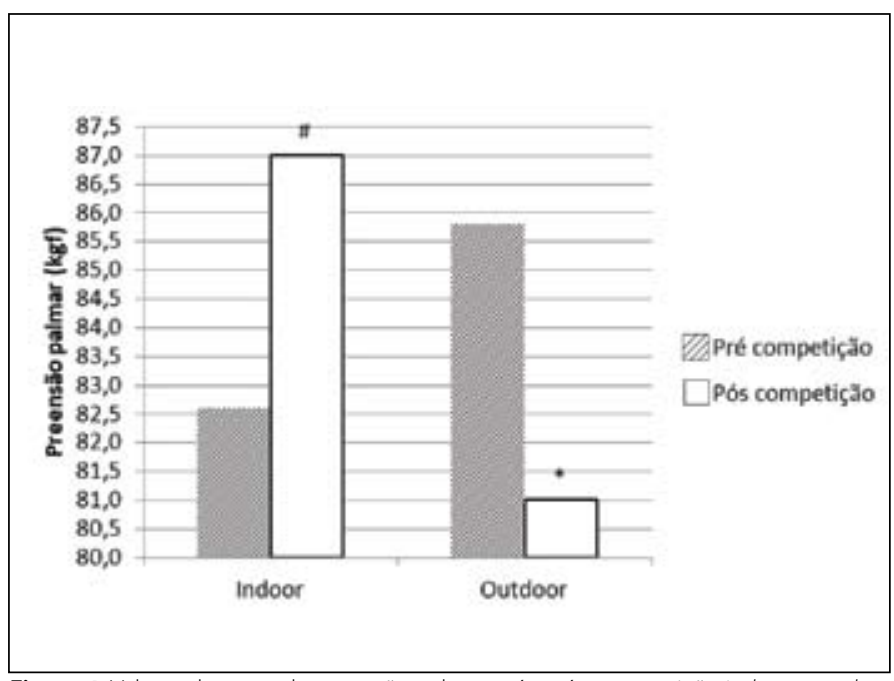

Figura 4. Valores de soma de preensão palmar pré e pós-competição indoor e outdoor de arco e flecha (\#* $\left.\#^{*} 0,05\right)$.

Os valores médios obtidos pela escala de Borg durante a competição indoor não apresentaram variação estatisticamente significativa, com classificação de 12,7 \pm 0,7 (leve - um pouco intensa) na escala de percepção de esforço para o período antes da prova e de 13,0 0,9 (um 
pouco intenso) para o período após a prova. No segundo dia de competição, outdoor, houve diferença significativa $(p<0,005)$ nos resultados obtidos pré e pós-competição, ficando entre 13,6 \pm 2,1 (um pouco intensa) e 16,4 0,8 (intensa - muito intensa), respectivamente.

\section{DISCUSSÃO}

Referente ao estado nutricional dos atletas de arco e flecha, os resultados indicaram excesso de gordura corporal. Estudos específicos sobre a composição corporal nesta modalidade esportiva são praticamente inexistentes, sendo o primeiro no contexto nacional. O percentual de gordura corporal variou amplamente entre 13,9 a 31,1\%.

A taxa de sudorese e a perda de peso corporal dos atletas sofreram variação significativa nos dois dias analisados, significativamente maior na competição outdoor, provavelmente devido às diferentes condições climáticas entre os dias. Quanto à taxa de sudorese indoor (1,5 $\pm 1,4 \mathrm{~L} / \mathrm{h})$ esta foi comparável a esportes como basquete $(1,6 \mathrm{~L} / \mathrm{h})$, futebol $(1,5 \mathrm{~L} / \mathrm{h})$, tênis $(1,6 \mathrm{~L} / \mathrm{h})$ e meia maratona $(1,5 \mathrm{~L} / \mathrm{h})$ entre outros ${ }^{(30)}$; já na outdoor $(2,2 \pm 1,3 \mathrm{~L} / \mathrm{h})$, poucos esportes se comparam ao volume de perda hídrica, sendo registrado apenas para futebol americano $(2,1 \mathrm{~L} / \mathrm{h})$ e squash $(2,4 \mathrm{~L} / \mathrm{h})^{(30)}$. Como consequência desta taxa de sudorese, principalmente no dia de competição outdoor (1,0 $\pm 0,2 \mathrm{~L})$, os atletas foram submetidos, segundo o $\mathrm{ACSM}^{(30)}$, a uma "desidratação leve", isto é, perda de peso entre 1 a 3\%; sendo que, mesmo neste grau, pode ocorrer um maior esforço cardiovascular, constatado através de um aumento desproporcional da frequência cardíaca durante o exercício, além de limitar a capacidade corporal de transferir calor dos músculos em contração para a superfície da pele, responsável pela dissipação do calor do corpo para o ambiente, provocando uma elevação na temperatura interna corporal (hipertermia) ${ }^{(31,32)}$. Por sua vez, a hipertermia pode diretamente prejudicar a performance motora pela redução da produção de força muscular, resultando em maior taxa de oxidação do glicogênio para a recuperação da capacidade funcional do sistema cardiovascular ${ }^{(33)}$.

Estes dados são reforçados pela avaliação da percepção subjetiva de esforço dos atletas, mensurada pela escala de Borg, na qual houve uma diferença significativa de percepção e esforço entre os valores iniciais e finais das competições indoor e outdoor, confirmando seu potencial como marcador de estresse imposto aos atletas pelas condições adversas ambientais ao ar livre ${ }^{(34,35)}$.

Apesar da ingestão de líquidos ad libitum durante os dias de competição, esta demonstrou ser insuficiente para evitar uma perda de peso significativa, fato também constatado em diversos estudos, nos quais atletas de forma voluntária não conseguem ingerir a quantidade necessária de líquidos para repor as perdas hídricas pelo suor, condição reconhecida como "desidratação involuntária"(36). A média de ingestão hídrica, tanto no dia de competição indoor (0,10 \pm 0,05L/h) quanto outdoor $(0,20 \pm 0,05 \mathrm{~L} / \mathrm{h})$, ficou muito abaixo do registrado para a maioria dos esportes, sendo comparável apenas com pólo aquático $(0,14 \mathrm{~L} / \mathrm{h})$ e meia maratona $(0,15 \mathrm{~L} / \mathrm{h})^{(30)}$.

Duas razões podem ser destacadas para estimular a ingestão de líquidos durante treinos e competições: a primeira é minimizar o efeito da desidratação e hipertermia e, deste modo, aumentar a efetividade e segurança do treinamento; a segunda refere-se à importância de se testar diferentes bebidas repositoras para se saber qual a mais eficaz para a reidratação de cada atleta ${ }^{(32)}$, sendo esta última uma extensão natural para futuros estudos com atletas de arco e flecha.

Notadamente, os valores de soma de preensão manual se comportaram antagonicamente nos dois dias de competição. Na competição indoor, foram observados valores de soma de preensão pós significativamente maiores do que os de pré-competição. Esta diferença pode ser atribuída ao aumento da temperatura corporal (valor não mensurado), especificamente da musculatura esquelética, durante a realização do exercício, que pode ter beneficiado a performance pelo aumento da velocidade das reações químicas, condução nervosa e mudanças conformacionais, condições próprias envolvidas na contração muscular ${ }^{(31)}$. Entretanto, na competição outdoor, houve redução significativa dos valores de soma de preensão pós-competição, indicando prejuízo na produção de força, que pode estar relacionado ao desproporcional aumento da temperatura corporal induzida pelas condições adversas ambientais. Já foi documentado o mecanismo adaptativo pelo qual há uma reação antecipada do organismo, para um menor recrutamento motor, com finalidade de antecipar os prejuízos advindos da hipertermia, mantendo assim a homeostase termal durante o exercício no calor ${ }^{(37)}$. Somado a hipertermia e suas consequências na geração de força, houve um quadro de desidratação significativo em relação à competição indoor, que pode ter atuado sinergicamente no aumento da temperatura corporal. Altas temperaturas corporais podem inibir a habilidade cerebral de realizar uma adequada condução neural para o músculo esquelético ${ }^{(38)}$.

Adicionalmente, Maughan ${ }^{(39)}$, em sua revisão sobre os possíveis efeitos da desidratação em atletas de alto nível de modalidades de força/potência, relata que uma perda hídrica entre 1,5 a 3\%, induzida por sauna (desidratação passiva) e restrição hídrica, respectivamente, tiveram impacto de redução de 6\% a 11\% na geração de força, resultados semelhantes ao encontrado para os atletas de arco e flecha na competição outdoor, cuja perda hídrica de 2,1 \pm 0,7\% resultou em redução de 5,6\% na força dada pelo teste de preensão palmar. Corroborando as hipóteses delineadas neste estudo, Nybo e Nielsen ${ }^{(35)}$ demonstraram aumento na dificuldade de manutenção da força durante exercício prolongado com progressiva hipertermia e desidratação, refletindo em aumento na percepção de esforço subjetivo mensurável.

Com relação à análise da glicose sanguínea antes e após as competições, não houve diferença estatística entre os valores, assim como entre os dois dias de competição. Verificou-se um aumento não significativo da glicemia pós-competição, que encontra explicação consoante ao trabalho de Keller et al. ${ }^{(40)}$ que investigaram os efeitos do estado de hidratação no metabolismo glicídico e evidenciaram que as concentrações de glicose plasmática aumentaram durante o estado de hiperosmolalidade, comparado à iso-osmolalidade, fato atribuído à indução da glicogenólise hepática. Judelson et al. ${ }^{(41)}$ verificaram também um aumento não significativo na glicose sanguínea antes e após exercício de resistência nos indivíduos que apresentaram uma perda média de peso de 2,5\%, sendo este aumento significativo apenas para o grupo que teve uma perda média de 5,0\% do peso corporal. Adicionalmente, análises da concentração de lactato e glicogênio muscular seriam necessárias para se avaliar corretamente o metabolismo energético envolvido na competição de arco e flecha e qual a predominância de utilização destes e outros substratos.

\section{CONCLUSÃO}

O presente estudo foi o primeiro a realizar avaliação antropométrica duplamente indireta em atletas brasileiros de alto nível de arco e flecha, além de determinar a taxa de sudorese, ingestão hídrica voluntária e perda hídrica em situação de competição indoor e outdoor. Considerando-se que os atletas foram expostos a diferentes condições climáticas nas competições indoor e outdoor, que reflete interessante experimento de condições ambientais adversas para a competição de arco e flecha, pôde-se constatar claramente o prejuízo no desempenho esportivo através da redução na força muscular. Embora utilizando um marcador bioquímico clássico de rendimento esportivo (glicemia), não foi constatada tal alteração nos resultados e na correlação com geração de força. Os biomarcadores mais sensíveis envolvendo arqueiros foram 
os relacionados à alteração aguda no estado hídrico, além da escala subjetiva de percepção de esforço. Como futuros desdobramentos experimentais, por se tratar de uma modalidade que exige alto grau de concentração dos atletas, estudos que avaliam as alterações no estado de humor e seu impacto na função cognitiva e rendimento devem ser realizados, para que as lacunas existentes em relação ao esporte sejam preenchidas.

\section{AGRADECIMENTOS}

À toda a equipe de arco e flecha, em especial ao técnico Reinaldo Augusto Nunes.

Todos os autores declararam não haver qualquer potencial conflito de interesses referente a este artigo.

\section{REFERÊNCIAS}

1. Ertan H, Kentel B, Tümer ST, Korkusuz F. Activation patterns in forearm muscles during archery shooting. Hum Mov Science. 2003;22:37-45.

2. FITA - International Archery Federation. Disponivel em: <http://www.archery.org >. Acesso em: 21 fev. 2008.

3. FPAF - Federação Paulista de Arco e Flecha: Tipos de competições. Disponível em: <http://www.fpaf. com.br. Acesso em: 21 fev. 2008.

4. Oppermann P. Ansiedade X Desempenho. Federação Paulista de Arco e Flecha. Disponível em: <http:// www.fpaf.org.br>. Acesso em: 14 abr. 2008

5. Midgley AW, McNaughton LR, Sleap M. Infection and the elite athlete: a review. Res Sports Med. 2003;11:235-59.

6. Patel AV, Mihalik JP, Notebaert AJ, Guskiewicz KM, Prentice WE. Neuropsychological performance, postural stability, and symptoms after dehydration. J Athletic Training. 2007;42:66-75.

7. Grandjean AC, Grandjean NR. Dehydration and cognitive performance. J Am Coll Nutr 2007;26 :549S-54S

8. Antunes HKM, Santos RF, Cassilhas R, Santos RVT, Bueno OFA, Mello MT. Exercício físico e função cognitiva: uma revisão. Rev Bras Med Esporte. 2006;12:108-14.

9. Cian C, Barraud PA, Raphael C, Jimenez C, Melin B. Influences of variations in body hydration on cognitive function: effects of hyperhydration, heat stress, and exercise-induced dehydration. Int $J$ Psychophysiol. 2000;14:29-36.

10. Wilson MG, Morley JE. Impaired cognitive function and mental performance in mild dehydration. Eur J Clin Nutr 2003;57:S24-9.

11. Judelson DA, Maresh CM, Yamamoto LM, Farrell MJ, Armstrong LE, Kraemer WJ, et all. Effect of hydration state on resistance exercise-induced endocrine markers of anabolism, catabolism, and metabolism. J Appl Physiol. 2008;105:816-24.

12. Timmons BW, Bar-Or O, Riddell MC. Oxidation rate of exogenous carbohydrate during exercise is higher in boys than in men. J Appl Physiol. 2003;94:278-84.

13. Sapata KB, Fayh APT, Oliveira AR. Efeitos do consumo prévio de carboidratos sobre a resposta glicêmica e desempenho. Rev Bras Med Esporte. 2006;12:189-94.

14. Achten JSL, Halson L, Moseley MP, Rayson A, Casey, Jeukendrup AE. Higher dietary carbohydrate content during intensified running training results in better maintenance of performance and mood state. J Appl Physiol. 2003;96:1331-40

15. Halyburton AK, Brinkworth GT, Wilson CJ, Noakes M, Bucley JD, Keogh JB, et al. Low- and hig-carbohydrate weight-loss diets have similar effects on mood but not cognitive performance. Am J Clin Nutr. 2007:86:580-7

16. Winnick JJ, Davis JM, Welsh RF, Carmichael MD, Murphy EA, Blackmon JA. Carbohydrate feedings during team sport exercise preserve physical and CNS function. Med Science Sports Exerc. 2005;37:306-15.

17. Rossi L, Tirapegui J. Implicações do sistema serotoninérgico no exercício físico. Arq Bras Endocrinol Metab. 2004;48:227-33.

18. Lane A. Relationships between perceptions of performance expectations and mood among distance runners: The moderating effect of depressed mood. J Science Med Sport. 2001;4:116-28.
19. Rossi L, Caruso L, Galante AP. Avaliação nutricional: novas perspectivas. ed Roca, 2009

20. Jackson AS, Pollock ML, Ward A. Generalized equation for prediction body density of women. Med Sci Sports Exerc. 1980;12:175-82

21. Jackson AS, Pollock ML. Generalized equation for predicting body density of men. Br J Nutr. 1978; 40:497-504.

22. Lohman TG, Roche AF, Martoreli R. Anthropometric standardization reference manual. Illinois: Human Kinetics Books, 1992.

23. Reis VAB, Azevedo COE, Rossi L. Perfil antropométrico e taxa de sudorese no futebol juvenil. Rev Bras Cineantropom Desempenho Hum. 2009;11:134-41.

24. Shirreffs SM. Markes of hydration status. Eur J Clin Nutr. 2003;57:S6-59.

25. ACSM (American College of Sports Medicine): Manual para avaliação da aptidão física relacionada à saúde. 1 ed. Rio de Janeiro: Guanabara Koogan, 2006.

26. Borg G. Escalas de Borg para a dor e o esforço percebido. 1 ed. Barueri: Manole, 2000

27. Yazbek JP, Mastrocolla LE, Negrão CE. Retorno à atividade física pós-tratamento cardiológico. In: Ghorayeb N, Barros T. O exercício. 1 ed. São Paulo: Atheneu, cap. 28, p. 305-12, 1999

28. ACSM (American College of Sports Medicine). Position stands: exercise and fluid replacement. Med Sci Sports Exer. 2007;268:377-90

29. Machado-Moreira CA, Vimieiro-Gomes AC, Silami-Garcia E, Rodrigues LOC. Hidratação durante o exercício: a sede é suficiente? Rev Bras Med Esporte 2006;12:405-9.

30. Rossi L, Reis VB, Azevedo CE. Desidratação e recomendações para a reposição hídrica em crianças fisicamente ativas. Rev Paulista de Pediatria, in press., 2010.

31. Pilcher JJ, Nadler E, Busch C. Effects of hot and cold temperature exposure on performance: a metaanalytic review. Ergonomics. 2002:45:682-98.

32. Nybo L. Hyperthermia and fatigue. J Appl Physiol. 2008;104:871-8.

33. Nybo L, Nielsen B. Perceived exertion is associated with an altered brain activity during exercise with progressive hyperthermia. J Appl Physiol. 2001;91:2017-23.

34. Sawka MN, Montain S. Fluid and electrolyte supplementation for exercise heat stress. Am J Clin Nutr. 2000;72(S1):564S-72S

35. Tucker R, Rauch L, Harley YXR, Noakes TD. Impaired exercise performance in the hear is associated with and anticipatory reduction in skeletal muscle recruitment. Eu J Physiol. 2004;448:422-30

36. Silva AEL, De-Oliveira FR, Gevaerd MS. Mecanismos de fadiga durante o exercício físico. Rev Bras Cineantropom Desempenho Hum. 2006:8:105-13.

37. Maughan RJ. Impact of mild dehydration on wellness and on exercise performance. Eur J Clin Nutr. 2003;57:S19-523.

38. Keller U, Szinnai G, Bilz S, Berneis K. Effects of changes in hydration on protein, glucose and lipid metabolism in man: impact on health. Eur J Clin Nutr. 2003;57:S69-S74.

39. Judelson DA, Maresh CM, Yamamoto LM, Farrell MJ, Armstrong LE, KraemerWJ, et al. Effect of hydration state on resistance exercise-induced endocrine markers of anabolism, catabolism, and metabolism. J Appl Physiol. 2008;105:816-24 